\title{
La gestión comunicacional: entre la precarización profesional y el diseño algorítmico
}

Notas sobre los desafíos actuales al quehacer de los comunicadores ante la tercera fase de la ciencia, el tercer espacio de aprendizaje y la tercera ola de las humanidades digitales, en un contexto de transformación digital.

The communication management: between professional precariousness and algorithmic design

A gestão da comunicação: entre precariedade profissional e projeto algorítmico

Carlos Eduardo Cortés S., Colombia

Director, Carrera de Comunicación Social

Facultad de Comunicación y Lenguaje

Pontificia Universidad Javeriana

Chasqui. Revista Latinoamericana de Comunicación

N. ${ }^{\circ} 141$, agosto - noviembre 2019 (Sección Monográfico, pp. 127-148)

ISSN 1390-1079 / e-ISSN 139o-924X

Ecuador: CIESPAL

Recibido: 03-o9-2019 / Aprobado: 22-11-2019 


\section{Resumen}

El quehacer de los comunicadores está inmerso de manera estratégica en cualquier actividad que involucre la conducta humana y las estructuras sociales. Pero el cambio de la era digital no es pertinente tan solo a los espacios disciplinares de la Comunicación Social. Vivimos en un ecosistema digital basado en una omnipresencia mediática en la cual usamos todos los medios en forma interconectada, en una sociedad-red -con su corolario de sociedadplataforma- que nos vuelve dependientes del diseño algorítmico ante una esfera pública global comercializada. Dicha esfera requiere cada vez más gestión de la comunicación pero, al mismo tiempo, desborda el quehacer profesional de los comunicadores, pues multiplica los gestores más allá de nuestro ámbito profesional. Los referentes analógicos del siglo XX resultan, pues, insuficientes para explicar y comprender el presente. Muchos de nuestros conceptos actuales tienen que mutar, porque los objetos y las prácticas sociales están cambiando desde finales del siglo XX, incluyendo la producción de conocimiento científico en las humanidades digitales, los procesos de enseñanza-aprendizaje y la transformación digital en que los antiguos empleados trabajan ahora como gestores independientes de proyectos, con la consecuente precarización del ámbito laboral. Una clave de futuro son la promoción y el acompañamiento del aprendizaje.

Palabras clave: economía política, información, comunicación, cultura, latinoamérica

\section{Abstract}

The task of communicators is strategically immersed in any activity that involves human behavior and social structures. But the change of the digital age is not only relevant to the disciplinary spaces of Social Communication. We live in a digital ecosystem based on a media omnipresence in which we use all media in an interconnected way, in a network-society - with its corollary of society-platform - that makes us dependent on algorithmic design before a globalized public sphere. This sphere requires more and more communication management but, at the same time, it overflows the professional work of the communicators, because it multiplies the managers beyond our professional field. The analogical references of the twentieth century are therefore insufficient to explain and understand the present. Many of our current concepts have to mutate, because social objects and practices are changing since the end of the 20th century, including the production of scientific knowledge in the digital humanities, the teaching-learning processes and the digital transformation in which former employees They now work as independent project managers, with the consequent precariousness of the work environment. A key to the future is the promotion and accompaniment of learning.

Keywords: political economy, information, communication, culture, Latin America 


\section{Resumo}

A tarefa dos comunicadores está estrategicamente imersa em qualquer atividade que envolva o comportamento humano e as estruturas sociais. Mas a mudança da era digital não é relevante apenas para os espaços disciplinares da Comunicação Social. Vivemos em um ecossistema digital baseado na onipresença da mídia na qual usamos todas as mídias de maneira interconectada, em uma sociedade em rede - com seu corolário de plataforma em sociedade que nos torna dependentes do design algorítmico diante de uma esfera pública globalizada. Essa esfera requer cada vez mais gerenciamento da comunicação, mas, ao mesmo tempo, transborda o trabalho profissional dos comunicadores, porque multiplica os gerentes além do nosso campo profissional. As referências analógicas do século XX são, portanto, insuficientes para explicar e entender o presente. Muitos de nossos conceitos atuais precisam sofrer mutações, porque os objetos e práticas sociais estão mudando desde o final do século XX, incluindo a produção de conhecimento científico nas humanidades digitais, os processos de ensino-aprendizagem e a transformação digital em que ex-funcionários Eles agora trabalham como gerentes de projeto independentes, com a consequente precariedade do ambiente de trabalho. Uma chave para o futuro é a promoção e acompanhamento da aprendizagem.

Palavras-chave: economia política, informação, comunicação, cultura, américa latina 


\section{Introducción}

Vivimos un tiempo que requiere no solo gestionar sino investigar la comunicación en medio de "pesimismo político, incertidumbre tecnológica y voluntad democrática”, al decir de Gabriel Kaplún (2018). Las nociones actuales de "sociedadplataforma" y "era de las plataformas" describen la inextricable relación entre plataformas digitales contemporáneas y estructuras sociales. Es decir, que dichas plataformas no reflejan lo social sino producen las estructuras sociales en que vivimos (Jorgensen, 2019; Van Dijck, et al. 2018), con los consecuentes resultados, positivos y negativos, para la vida democrática del planeta, en general, y de América Latina y el Caribe, en particular.

A pesar de los ideales de la internet temprana como "comunal electrónico", los algoritmos con que funcionan las plataformas digitales no fueron creados bajo criterios de respeto a los derechos humanos, el bien común o la dignidad humana. Su motivación central ha sido, y sigue siendo, el lucro corporativo: un puñado de empresas privadas (Alphabet Google, Amazon Apple, Facebook, Microsoft $y$ Twitter), media y re-media la mayor parte de nuestro tiempo en línea, regido por un mercado cuya mercancía es la atención humana, mientras los proveedores de internet administran la infraestructura pública basada en la web, con un resultado alarmante para la vida social: una esfera pública global comercializada que requiere cada vez más gestión de la comunicación pero, al mismo tiempo, desborda el quehacer profesional de los comunicadores.

Se trata, pues, de retos descomunales que todavía no son suficientemente atendidos en nuestro campo. En palabras de Pons (2013),

en medio de la transformación digital, zarandeados por el remolino del cambio, la sensación de confusión y desconcierto es inevitable para comprender algunos de los fenómenos que acompañan al 'desorden digital' en el que estamos inmersos, [incluyendo] los cambios en los soportes, en la lectura, en la escritura, en el documento y el archivo mismos, en la condición y la función de autor, en los modos de colaboración y en la difusión del conocimiento.

La sociedad-plataforma no solo multiplicó los gestores de comunicación, sino que la ausencia de la academia en numerosos espacios sociales, no ha significado que las necesidades de comunicación no hayan sido cubiertas, pues "la revolución digital ha puesto en crisis la vieja monopolización de la emisión, los recursos están a la mano, bastan la conectividad y la iniciativa" (Prieto Castillo, 2016).

Nuestra gestión no logra, pues, administrar esta difícil etapa en que estamos sumidos, porque el lastre anacrónico de referentes analógicos resulta insuficiente para explicar el presente. En otras palabras, muchos de nuestros conceptos actuales tienen que mutar, porque los objetos y las prácticas sociales están cambiando desde finales del siglo XX (Cortés, 2014b. En Piscitelli, 2008). 
Incluso, pese a referirnos con frecuencia a las tecnologías de información y comunicación (TIC), aún hoy no manejamos con claridad la distinción entre una y otra, ni cuándo nos referimos a TIC analógicas o a TIC digitales. Más aún, la pregunta sobre “¿Qué es comunicación?” no solo nos ha acompañado desde la década de 1960, sino que en 2016 volvió a ser el tema central de un evento académico en Brasil (Peruzzo, 2018).

Como gestor de proyectos de comunicación estratégica, y de diseño y desarrollo de contenidos (educativos, informativos y de entretenimiento), mi ejercicio profesional me permitió convertirme en un observador privilegiado durante una experiencia acumulada de trabajo en universidades, organismos estatales, no gubernamentales, entidades internacionales y medios, tanto analógicos como digitales, en Latinoamérica, Estados Unidos, África y Asia. En consecuencia, mi propósito es señalar algunas pistas percibidas en nuestro quehacer comunicacional, pues la "transformación digital" nos convoca a todos si la entendemos como "ser más adaptables al cambio en sí mismo" (Rust, 2018). Se trata del uso de las TIC digitales y las nuevas formas de trabajo que ha generado esta era de las plataformas, para comprender el cambio que se está produciendo en cualquier organización humana. El objetivo es ser capaces de actuar de manera mucho más dinámica y rápida de lo que las organizaciones lo han hecho antes.

\section{El alcance de la gestión}

El quehacer de los comunicadores está siempre inmerso de manera estratégica en el centro de cualquier actividad que involucre la conducta humana y las estructuras de las sociedades, en especial si se considera el sentido mismo de la palabra "gestión", cuya raíz es el verbo latino "gerere", que significa "hacer". "Gerente" y "gerencia" vienen de la misma raíz y se conectan con otros verbos como administrar, gobernar, dirigir y regular. La palabra proviene de "gestioonis": acción de llevar a cabo y, además, está relacionada con "gesta", en tanto historia de lo realizado, y con "gestación", llevar encima (Huergo, 2007).

Nuestra gestión, como espacio del hacer y del logro de resultados, permite hoy repensar toda la teoría estratégica desde la comunicación (Arribas, et al., 2017), de manera que la lógica de la gestión de proyectos opera hoy como una base de aprendizaje destinada a aplicar conocimientos, habilidades, herramientas y técnicas para diseñar actividades que cumplan con los requisitos de un emprendimiento temporal que planifica participantes con sus funciones, entregables, fechas límite, presupuesto, recursos, ejecución, monitoreo y cierre del proceso. En tal sentido, la gestión comunicacional tiene hoy, en la lógica de proyectos, un instrumento útil para abordar cualquier esfuerzo de comunicación que integra áreas, programas y recursos de comunicación e información para:

- identificar y perfilar destinatarios, beneficiarios o grupos de interés; 
- producir contenidos multi-plataforma;

- elegir canales y tácticas de comunicación (actividades y materiales);

- establecer formas de cooperación y asociación con otros individuos u organizaciones;

- implementar acciones de corto, mediano y largo plazo, y

- evaluar y corregir el curso del proyecto cuando resulta necesario.

Bajo el potencial de esa lógica, cualquier buena gestión se sostiene en tres pilares:

1. Planificación: procedimientos que nos permiten alcanzar determinadas metas y objetivos para organizar nuestras actividades y usar eficientemente nuestros recursos.

2. Diagnóstico: reconocimiento de la situación actual. Diferencia los elementos de una realidad en términos positivos y negativos (fortalezas y debilidades) para detectar causas de los problemas y decidir vías de acción para irlos resolviendo. Se centra en el pasado (de dónde venimos) y el presente (dónde estamos).

3. Prospectiva: anticipación para actuar en el propio contexto con base en una idea de futuro que nos permite ir hacia adelante al aprovechar potencialidades, proyectar alternativas y definir rutas para cambiar la realidad en un sentido deseado (para dónde vamos).

Sin embargo, la noción misma de gestión "se ha ampliado y se ha hecho más compleja, para responder a la propia complejidad creciente del entorno social: gestionar no es exclusivamente administrar (o 'gerenciar'), ni simplemente organizar y, mucho menos, conducir (en sus sentidos autocrático, carismático, paternalista, etc.). Tampoco es algo que siga la lógica de las 'recetas', tantas veces relacionadas con el marketing estratégico o la producción de imagen corporativa" (Huergo, 2007). Por el contrario, la gestión de la comunicación se ha desplegado en diversos ámbitos que la requieren, desde la gestión del discurso, de la escucha y de las redes, pasando por la gestión en situaciones de comunicación, hasta la gestión de la memoria y de las comunidades de aprendizaje, tanto desde lo individual como desde lo institucional (Prieto Castillo, 2016).

De allí que la mayor complejidad genere una tensión fundamental en el campo comunicacional: nuestra dificultad histórica para combinar de manera realmente interdisciplinaria los aspectos científicos, educativos y profesionales responsables del avance académico del campo, con las consecuentes debilidades en nuestra gestión, en general. 


\section{Una débil gestión de la investigación}

Si admitimos que la teoría es básicamente una herramienta de investigación, no su resultado final (Castells, 2000), en la actualidad deberíamos estar en mejor posición para investigar nuestro hacer y apoyarlo desde el propio proceso académico y de enseñanza-aprendizaje a partir de una constatación de fondo: la comunicación no es un simple conducto neutral para transmitir información, sino el proceso social primario por medio del cual se construye nuestro mundo significativo común (Craig, 2009),

A la luz de la llamada tercera fase de la ciencia, como lo explica Harvey (2017),

Debemos reconocer el rol fundamental de la comunicación no solo a nivel de ciertos asuntos humanos, sino en el conjunto de la cultura y el ambiente. [...] Los medios y las redes sociales son ante todo redes de comunicación y de conversación globales que ponen en juego lenguajes simbólicos, manifestaciones culturales, relaciones de poder, consensos globales sobre la responsabilidad y el diseño de ambientes virtuales al servicio del codiseño de ambientes complejos. [...] La separación del observador y del objeto en la ciencia se basa en conceptos imaginativos restrictivos y reductores de la complejidad de los sistemas vivientes, tal como la realizaba la primera fase de la ciencia. En la segunda fase, hemos aprendido a plantear mejores preguntas y a construir mejores objetos [...] pero había que ir más allá.

Nuestra época favorece la emergencia colectiva de una tercera fase de la ciencia, por la integración de múltiples puntos de vista, la transdisciplinariedad en los partenariados de investigación y el desarrollo complejo, y el compromiso de los usuarios y ciudadanos en nuevos procesos de producción de conocimientos. [...] Las comunidades colaborativas en ascenso en la web son los actores de esta tercera fase de la ciencia, en la que el objeto es observado según una dialéctica de confrontación/tensión/equilibrio entre los puntos de vista realistas, subjetivistas e individuales, por una parte, y socioconstructivistas, colectivos e interaccionistas, por otra. Se observa en esos nuevos contextos plurales y comunicacionales la explosión de diversos modos de lenguaje y de 'relatos científicos interconectados', en los que la interdependencia recíproca de millones de usuarios/diseñadores permite compartir perspectivas transdisciplinares, transculturales, transprofesionales en torno a problemáticas de interés global. (Harvey, 2017.pp. XXXXXXII)

Un antecedente de esas formas de concebir y practicar la comunicación se produjo en los años 1980, como una valiosa aproximación teórico-práctica a la gestión comunicacional: con el auspicio de la Fundación Friedrich Ebert, Ciespal dio respuesta a una demanda de las escuelas y facultades de Comunicación Social de aquel entonces, mediante un curso intensivo de planificación de proyectos de comunicación dirigido a profesores latinoamericanos, a cargo de Daniel Prieto 
Castillo y Eduardo Contreras, para capacitarse en diagnóstico de comunicación, evaluación comunicacional, planificación de la comunicación en las relaciones internas y externas, análisis de mensajes, comunicación interpersonal y basada en materiales (Prieto Castillo, 1998).

Dos elementos centrales surgieron de ese proceso: el primero, la posibilidad de planificar la comunicación sin los sesgos economicistas propios de las ciencias sociales de la segunda posguerra, tan presentes en los esfuerzos del desarrollismo y la teoría de la dependencia en los años 1960 y 1970 . Y el segundo, el despliegue de una dimensión poderosa y perdurable: la "comunicación educativa", entendida como:

una forma de acompañamiento a procesos sociales que tome en cuenta no lo que inducimos a hacer a través de la comunicación, sino lo que logramos en aprendizajes en las relaciones presenciales o bien mediante las posibilidades ofrecidas por los medios tradicionales y las nuevas tecnologías de la información y de la comunicación, incluyendo el autodiagnóstico comunitario. (Prieto Castillo, 1998)

Desde aquellos años había una gran fortaleza para la historiografía de los campos académico y científico de la comunicación, la mirada sobre el propio campo y el desarrollo de teorías y metainvestigaciones en comunicación en América Latina (Ferreira \& Peruzzo, 2018). Pero, respecto al reducido repertorio de métodos y técnicas que exhibimos como profesores investigadores, nos agobia hasta el presente una serie de pronunciadas debilidades en la gestión misma de la investigación latinoamericana de la comunicación.

De hecho, dicha gestión se mantiene,

en una situación de marginalidad entre las ciencias sociales (una especie de 'hermanita menor' a la que se trata condescendientemente) [mientras] sostiene una serie de tensiones que se originan en una múltiple desvinculación entre enseñanza e investigación; entre investigación y campos profesionales; entre enseñanza y campos profesionales; entre investigación básica e investigación aplicada. (Sánchez Ruiz, 2003)

Y se trata de tensiones que Maria Immacolata Vassalo de Lopes rastrea hasta la primera década del siglo XXI, debido a deficiencias como:

- ausencia de reflexión epistemológica;

- insuficiente dominio de teorías (debilidad teórica), y de la interdisciplinariedad;

- falta de visión integrada de la teoría (nivel teórico más técnico);

- deficiente combinación de métodos y técnicas;

- énfasis en investigación descriptiva,y 
- dicotomía entre investigaciones cuantitativas y cualitativas (Lopes, 2004, En Peruzzo, 2018).

Para Marques de Melo la Escuela Latinoamericana de Comunicación no se entregó,

a la tarea de solamente hacer investigaciones para el mercado en el sentido de solucionar problemas de las empresas ni tampoco quedarse alejada del mundo concreto como muchas veces algunos europeos han intentado hacer, intentando no mezclarse con la realidad. Los latinoamericanos buscaron temas de investigación, objetos de investigación, que tenían interés para nuestras sociedades y nuestras comunidades. (Marques de Melo, 2000. En: Peruzzo, 2018)

No obstante, en 2009, Martín-Barbero y Rincón se preguntaban cuáles de nuestros saberes resultan desechables y cuáles son realmente indispensables, para poder asumir,

otra posición, arriesgada pero responsable, que busca el lugar-entre [...] el 'claustro' universitario y el torbellino social, una de cuyas figuras es la que emerge en el entrecruzamiento de nuestras académicas, y con frecuencia inertes, líneas de investigación y una mínima agenda de país que desestabilice nuestros narcisismos e inercias obligándonos a mirar de frente ese afuera cuya realidad a la vez que nos sostiene - laboral e intelectualmente - nos desafía radicalmente. (MartínBarbero \& Rincón, 2009)

Y ese desafío, laboral e intelectual, resurge con fuerza no solo para exigir una investigación relevante o "de interés público" (Donsbach, 2012), sino para responder a la legítima necesidad de nuestros egresados de obtener un empleo digno para vivir de su profesión. Y entre los cambios más drásticos originados en la "plataformización" se encuentra, precisamente, el empleo.

\section{La precarización profesional}

El Estudio Económico y Social Mundial 2018, del Consejo Económico y Social de las Naciones Unidas, analizó los avances en las tecnologías de vanguardia o de punta - automatización, robótica, vehículos eléctricos, energía renovable, biotecnología e inteligencia artificial-, así como su impacto económico, social y ambiental. Y concluyó que, si bien dichas tecnologías tienen un potencial inmenso para promover crecimiento, prosperidad y sostenibilidad, y acelerar el logro de la Agenda 2030 para el Desarrollo Sostenible, también presentan nuevos y particulares desafíos de orden ético y moral, mientras nos enfrentan al riesgo de desempleo, subempleo e inequidad crecientes (Ecosoc, 2018).

El proceso de producción en el llamado "capitalismo digital" asume una estructura de red en la cual las organizaciones ya no poseen todas las partes de 
la cadena productiva sino que las asignan a otras compañías cuando y según lo vayan necesitando. El teletrabajo y el empleo circunscrito a proyectos son cada vez más comunes, mientras que la tendencia es que los antiguos empleados trabajen ahora como "gestores independientes de proyectos", de manera que se responsabilicen no solo de prestar servicios sino también de la comunicación y la coordinación de dichos servicios.

La nueva figura es una individualización o singularización exacerbada que está muy lejos del empleo remunerado y los beneficios logrados con las conquistas laborales del siglo XX. Por una parte, el emprendimiento otorga mucha más autonomía y creatividad que en la producción industrial del siglo pasado; pero, al mismo tiempo, es inseguro e incierto pues, en el siglo XXI, el trabajador nunca sabe si tendrá otro contrato, ni cuándo (Cortés, 2014b. En Siapera, 2012), de manera que se producen retrocesos a condiciones laborales propias del siglo XIX.

Como resultado, así como la empresa ya no está representada por una gran fábrica de producción masiva, de igual manera el empleo ya no se caracteriza por la permanencia ni por la estabilidad. Al contrario, la "flexibilización" generalizada tiende a ser la característica clave del trabajo actual, a medida que se ingresa en procesos de "plataformización" digital en los cuales entidades como Uber, Rappi o Deliveroo, entre otras, generan una precarización constante de los empleados y, a la vez, experimentan rechazos de sus trabajadores y nuevas formas de organización y sindicalización (Chen, et al., 2019).

De hecho, la implementación actual de todo tipo de plataformas digitales en la sociedad detona una feroz discusión acerca del beneficio privado y el lucro corporativo versus los intereses públicos y los beneficios colectivos. Se trata de una discusión pendiente porque la influencia de muchas plataformas ha crecido de manera desmedida, antes que un verdadero debate sobre valores públicos y bienes comunes pudiera comenzar (Van Dijck, et al., 2018). Como lo analiza la Organización Internacional del Trabajo (OIT), las plataformas para labor digital crean la oportunidad del teletrabajo desde cualquier lugar, en cualquier momento, para asumir cualquier contrato que resulte apropiado. Pero, hay riesgos asociados en relación con estatus de empleo, ingresos adecuados, protección social y otros beneficios, que ponen en cuestión la mínima decencia de una determinada labor (Berg, 2018).

En palabras de Couldry \& Rodríguez (2018):

La dependencia cada vez mayor que tienen los individuos de una infraestructura virtual que medie la vida cotidiana acrecienta la importancia de las compañías que proveen dicha infraestructura. Esta circunstancia ha transformado la gobernanza de las infraestructuras mediáticas, de formal a informal, y la importancia de instituciones y prácticas de gobernanza transnacional mediante las cuales son las empresas, y no los Estados, las que ejercen una influencia predominante incluso mediante la operación de algoritmos, con implicaciones ambiguas para el poder corporativo y los derechos individuales, la esfera pública y el progreso social [ante 
la] aparición de una economía de la información en red y la globalización de los flujos de información mediada.

\section{El "plegado" digital de la realidad}

Aunque las costumbres sociales, las estructuras legales y las prácticas empresariales que gobiernan la vida cotidiana hayan evolucionado a lo largo de centenas de años, y dicho desarrollo prosiga, hoy vivimos la transición de dichas realidades al ciberespacio como nuevo lugar de encuentro e interacción radicalmente diferente del mundo físico, en el cual las plataformas y las aplicaciones para redes digitales se combinan con una conectividad ubicua que libera las comunicaciones, las transacciones y otras interacciones, de sus limitaciones físicas (Windely, 2005), creando así todo un conjunto nuevo de requisitos y procedimientos que debilitan o cuestionan las visiones tradicionales de la gestión comunicacional tradicional.

Cuando "la mediación tecnológica de la comunicación deja de ser meramente instrumental para espesarse, densificarse y convertirse en estructural" (MartínBarbero, 2002a), el tercer espacio de aprendizaje (Borgnakke, 2012 : 2009), resulta muy útil para explicar la fusión del mundo físico (primer espacio), con el virtual (segundo espacio), en un lugar interconectado por redes que puede ser habitado por usuarios en forma remota, simultánea o asíncrona. Dicho espacio se caracteriza por ser poli-contextual y multivocal, y asume que las culturas son híbridas de manera inherente, porque contienen dimensiones y partes heterogéneas con orígenes disímiles e historias diferentes (Vistrain, 2009), de manera que la propia gestión educativa está siendo repensada a la luz de esa complejidad, pues la coherencia entre objetos, problemas e instrumentos de investigación educativa y cultura digital —en contextos que usan TIC digitales tales como entornos virtuales de aprendizaje (EVA), mundos digitales virtuales en $3 \mathrm{D}$, y redes sociales-, genera dudas epistemológicas, metodológicas y teóricas que todavía no se debaten de manera suficiente (Schlemmer, et al., 2013).

Estarelación tan estrecha entrelainvestigación humanísticaylacomputación ha dado lugar a las "humanidades digitales" para dar cuenta de la plasticidad de las formas digitales y la manera en que estas apuntan a una nueva forma de trabajar con representaciones y mediaciones, que podemos considerar como el "plegado" digital de la realidad, por lo que es posible acercarnos a la cultura de una manera radicalmente nueva: a fin de mediar un objeto, un dispositivo digital o computacional requiere traducirlo a código digital comprensible. Y sin dicha codificación el dispositivo no tiene un objeto para procesar (Berry, 2011).

Sin embargo, la traducción implica un recorte en el que una parte de la información debe descartarse para poder almacenar una representación dentro del dispositivo. En otras palabras, la computación requiere que todo se transforme desde el flujo continuo de nuestra realidad cotidiana en una 
matriz digital almacenable como representación de la realidad, la cual puede manipularse mediante algoritmos. Esos métodos sustractivos para entender la realidad (episteme), producen nuevos conocimientos y métodos para el control de la realidad (techné). Y lo hacen mediante una mediación digital que las humanidades digitales asumen como su problemática (Berry, 2011).

En tal sentido, la transformación de las humanidades y las ciencias sociales va más allá de su implicación con las TIC digitales, pues no se trata solo de una construcción de herramientas computacionales aplicada, sino del devenir de la propia computación como dominio científico con resultados teóricos acerca de la información y su transformación, la gestión de macrodatos (Big Data), algoritmos para transformar la información, y una gran cantidad de asuntos interdisciplinarios que incluyen interacciones con otros dominios, desde inteligencia artificial y robótica, hasta construcción automatizada, interfaces cerebro-computador, computadores cuánticos y biológicos, y redes sociales (Rosembloom, 2012).

De ahí que hoy se describa el "giro computacional" (computational turn), como una tercera ola de las humanidades digitales: código y software para entender dichas humanidades, en particular, y la producción cultural, en general, con implicaciones de largo alcance para las disciplinas, la pedagogía y nuestra gestión comunicacional (Berry, 2012).

Por ejemplo, un reto inmediato es determinar hasta dónde los enfoques computacionales del sonido y las imágenes amplían o cambian nuestra comprensión conceptual y epistemológica de los medios, en un momento en que los datos audiovisuales están en todas partes, desde fotografías, pasando por videos, videojuegos y deportes electrónicos, hasta las realidades mixta, aumentada y virtual, que incluyen (ro)bots de inteligencia artificial, piratería y formatos híbridos interactivos basados en la web y se encuentran en un devenir permanente, con nuevas fronteras de tecnologías digitales como la "Deepfake", que se basa en inteligencia artificial para producir o alterar el contenido.

En el camino de gestionar la comunicación en el ámbito educativo fuimos viviendo la complejidad creciente de la tecnicidad digital en nuestro quehacer (Cortés, 2004), que luego desembocaría en esta tercera ola de las humanidades digitales. En la década de 1990 la combinación interdisciplinaria de aspectos científicos, educativos y profesionales emergió a partir de la pregunta por el aprendizaje.

\section{La mediación del aprendizaje}

En 1990, Peter Senge le dio un vuelco a la teoría estratégica al advertir un obstáculo a la resolución de las crisis debido a nuestra propia manera de percibir el mundo: 
Desde muy temprana edad nos enseñan a analizar los problemas, a fragmentar el mundo. Al parecer esto facilita las tareas complejas, pero sin saberlo pagamos un precio enorme. Ya no vemos las consecuencias de nuestros actos: perdemos nuestra sensación intrínseca de conexión con una totalidad más vasta. [Hay que] destruir la ilusión de que el mundo está compuesto por fuerzas separadas y desconectadas. Cuando abandonamos esta ilusión podemos construir 'organizaciones inteligentes', organizaciones donde la gente expande continuamente su aptitud para crear los resultados que desea, donde se cultivan nuevos y expansivos patrones de pensamiento, donde la aspiración colectiva queda en libertad, y donde la gente continuamente aprende a aprender en conjunto" (Senge, 1994).

Al finalizar la década de 1980, el proyecto Radio Nederland Training Centre (RNTC), consolidado en la sede de Ciespal, se trasladó a San José de Costa Rica y logró conjugar lo que venían trabajando en Quito: la planificación, con instancias de investigación y producción de materiales. RNTC se involucró de lleno en la capacitación de:

- comunicadores para el medio rural mediante un convenio con el Instituto Interamericano de Cooperación para la Agricultura (IICA);

- comunicadores dedicados a la niñez, la juventud y la mujer, en un proyecto con UNICEF;

- organizaciones no gubernamentales comprometidas con el medio ambiente, con los derechos humanos y con la búsqueda de alternativas para la pobreza en la región;

- universidades y otros centros educativos y,

- sistemas de educación a distancia muy presentes en América Central y el Caribe (Prieto Castillo, 1998).

Dentro del proyecto de sintetizar el esfuerzo de la comunicación educativa, a la luz de todas las experiencias desarrolladas por RNTC, Daniel Prieto Castillo y Francisco Gutiérrez desarrollaron el concepto de "mediación pedagógica" como la tarea de promover y acompañar el aprendizaje desde las relaciones con la institución, con el educador, con los materiales, con el grupo, con el contexto y consigo mismo (Gutiérrez \& Prieto Castillo, 1990).

La "organización que aprende", tanto desde la mirada de Senge, como la de Prieto Castillo y Gutiérrez, constituyó una pista esencial para reorganizar la propuesta pedagógica del Servicio Conjunto de Comunicación, que agrupaba las organizaciones católicas de comunicación UNDA-AL, OCIC-AL y UCLAP (hoy en día, Signis, ALC). Con Daniel Prieto Castillo, Ana María Lalinde, Fernando López, y un equipo de comunicadores con sede en Quito, elaboramos una colección modular de educación a distancia en producción radiofónica y comunicación impresa. Los materiales fueron trabajados con más de 200 grupos de comunicadores populares en distintos países de la región y se produjeron con 
base en las propuestas de la mediación pedagógica, bajo la premisa de aprender a aprender como equipo de trabajo, y su correlato metacognitivo: enseñar a aprender, mediante una combinación de autoaprendizaje e interaprendizaje a distancia y presencial (Prieto Castillo, 1998).

Con esas bases, entre 2004 y 2006, de vuelta al laboratorio pedagógico de RNTC, Prieto Castillo \& Van de Pol elaboraron la noción de "aprendizaje ensanchado" para referirse al "e-Learning" como la posibilidad de ampliar el entorno de enseñanza-aprendizaje más allá de sus tradicionales límites físicos, geográficos y temporales, mediante el uso de tecnologías de información y comunicación (TIC) digitales en red (Prieto Castillo \& Van de Pol, 2006). De hecho, la UNESCO propuso como uno de los requisitos básicos para la educación del siglo XXI preparar a las personas para participar en una economía basada en conocimiento, incluyendo las perspectivas social y cultural. Y para ello, indicó el "e-Learning" como una piedra angular para construir sociedades del conocimiento inclusivas (Kozma \& Isaacs, 2011; Bates, 2001).

No hablamos, pues, de tecnologías educativas a secas, sino de la manera en que usamos dichas tecnologías para promover y acompañar aprendizajes. El “aprendizaje ensanchado" puede entenderse, así, como la intersección actual entre seres humanos, procesos educativos y tecnologías puestas al servicio del aprendizaje, en cualquier momento y lugar (United Nations University, 2008), porque vincula el aprendizaje individual y el organizacional mediante los conceptos de "inteligencia colectiva" y "conocimiento conectivo", y lo expresa en los nuevos medios sociales (Cortés, 2012; Yang \& Yuen, 2010). Tal como lo sugiere la teoría del "conectivismo", en nuestra realidad contemporánea:

- Tanto las organizaciones como los individuos aprenden.

- La creciente atención a la gestión del conocimiento resalta la necesidad de una teoría que intente explicar el vínculo entre el aprendizaje individual y el organizacional.

- Las herramientas que usamos definen y dan forma a nuestro pensamiento.

- Muchos de los procesos que antes manejaban las teorías del aprendizaje (en especial el procesamiento cognitivo de información), pueden ser ahora habilitados o apoyados por TIC digitales que "alteran" (recablean) nuestro cerebro.

- Elsaber-cómo (know-how)yelsaber qué(know-what), son complementados por el saber-dónde (know-where), en tanto comprender dónde hallar el conocimiento requerido (Siemens, 2005).

En otras palabras, una cultura de cambio, como la que vivimos con mucha intensidad, implica ajustar la mentalidad, los valores y el comportamiento dentro de cualquier organización humana. De allí que hoy resulte tan urgente reconsiderar muchas de nuestras premisas explicativas de la gestión, porque 
solo una "organización inteligente" puede enfrentar con solvencia el desafío del cambio producido por la "transformación digital".

Durante buena parte del siglo XX, el aprendizaje se enfocó en la adquisición de habilidades o la transmisión de información, en lo que se ha llamado "aprender acerca de". En la década de 1990, algunas teorías comenzaron a valorar el "aprender a ser", de manera que colocaron el aprendizaje en un contexto situacional vinculado tanto con sistemas e identidad, como con la transmisión de conocimiento. Y surgieron otras tipologías centradas en "aprender haciendo", "aprender interactuando" y "aprender compartiendo".

Esas modalidades funcionaron bien en la relativa estabilidad del siglo XX. No obstante, en el siglo XXI ya no resulta suficiente este abordaje: en un mundo en flujo constante necesitamos una teoría del "aprender a devenir" (learning-to-become):

Allí donde la mayoría de teorías del aprendizaje entienden devenir como un estado de transición para llegar a ser algo, queremos sugerir que el siglo XXI requiere que pensemos en el aprendizaje como una práctica de devenir una y otra vez. (Thomas \& Brown, 2009, p. 1)

Arribas (2017), lo expresa como "la transformación del hombre racional a hombre relacional en un sistema orgánico y aprendiente". Es decir, introduce la llamada "nueva teoría estratégica" como una concepción que genera la multidimensionalidad y la transdisciplinariedad necesarias para aceptar la incertidumbre y el caos como un síntoma de vitalidad capaz de llevar a cabo la gestión desde la complejidad. Por su parte, Fernández Collado sintetiza los siete cambios que Rafael Pérez propone como "nueva teoría estratégica" para mejorar la gestión tradicional (Pérez \& Massoni, 2009):

1. trabajar con los paradigmas actuales de la ciencia y no con los del siglo XVII;

2. recuperar al ser humano como clave central,

3. sustituir la concepción de la organización como racional y jerárquica, y pasar a otra sistémica, conectiva y socialmente responsable;

4. proporcionar reglas hermenéuticas y articuladoras en vez de paradigmáticas y exactas;

5. refundar la estrategia desde la comunicación, dándole una orientación más cooperativa y dialogante;

6. usar nuevos métodos de análisis estratégicos para entender las realidades sociales sin fragmentarlas o destruirlas, y

7. establecer nuevos modelos en la metodología que repliquen los procesos mentales humanos y eviten la artificiosidad de los modelos convencionales (Fernández Collado, prólogo en Arribas et al., 2017). 
Massoni y sus colaboradores, desde la escuela de Comunicación Estratégica de Rosario, Argentina, aplican esos principios en un proyecto que asume la comunicación como un fenómeno complejo, situacional y fluido, y considera las estrategias de comunicación como algoritmos fluidos cuyos indicadores comunicacionales(comolasdimensionesinformativa,interaccionaleideológica), constituyen un aporte diferencial respecto de las mediciones tradicionales de la comunicación, pues permiten entender la comunicación estratégica como una metaperspectiva centrada en el cambio social conversacional y abordan la multidimensionalidad de lo comunicacional en tanto encuentro en la diversidad, de manera que permiten desplegar nuevas competencias para el comunicador social, redefiniendo su tarea, tanto académica como profesional, en términos de encuentro sociocultural (Massoni, et al., 2019).

\section{Conclusión}

La nueva estructura de las relaciones humanas es la sociedad-red, con su corolario de sociedad-plataforma, basada en una estrecha vinculación entre cultura y fuerzas productivas, entre espíritu y materia, la cual ha permitido el surgimiento de nuevas formas de interacción, control y cambio social. A fines del siglo XX, este nuevo paradigma informacional - constituido alrededor de la microelectrónica, las TIC digitales, y la biotecnología- sustituyó o subsumió el paradigma previo del industrialismo - organizado en torno a la producción y distribución de energía - y hoy afecta, en mayor o menor medida, todas las sociedades del mundo, con diversas manifestaciones culturales e institucionales cuyas características son tanto positivas como negativas (Castells, 2006; 2001; 2000; 1997), porque conviven en un mundo conectivo en el cual las plataformas han penetrado el corazón de las sociedades - afectando instituciones, transacciones económicas y prácticas sociales y culturales-, de manera que han forzado a gobiernos y Estados a ajustar sus estructuras legales y democráticas (Van Dijck, 2018).

En ese sentido, es probable que nuestro mayor desafío para la gestión comunicacional es que nos encontremos en "el fin de la luna de miel digital", al decir de Cristóbal Cobo, porque,

La actual concentración del poder digital en unas pocas compañías [...] no solamente está generando nuevas formas de poder y control que exacerban las ya existentes, sino que además crea nuevas formas de exclusión y periferia, pero aun así, y evitando reduccionismos, dependiendo de la ruta que uno elija, puede transitarse desde el optimismo (utopía) a la tragedia (distopía), o simplemente desde el diagnóstico a la reacción (posible) [...]. No hay recetas ni doctrinas que puedan instalarse de manera tan sencilla como una actualización en su sistema operativo. El objetivo último es reflexionar desde una perspectiva crítica y abierta sobre las consecuencias de la masificación de las tecnologías y su impacto en las nuevas formas de poder y control de la sociedad actual. (Cobo, 2019) 
El hecho es que el cambio contextual de la era digital no está aislado ni es pertinente tan solo a los espacios disciplinares de la Comunicación Social. Vivimos en un ecosistema de comunicación digital basado en una omnipresencia mediática en la cual usamos todos los medios en forma interconectada (hipermedios y multimedios), para crear, almacenar, intercambiar y usar contenidos en el ciberespacio (con sus flujos de información y su cultura de convergencia basada en TIC digitales) (Jenkins, 2007 ;2006), de manera que, en dicho ecosistema, nos volvemos dependientes del diseño algorítmico porque las interfaces digitales forman una red que semeja un hipertexto expansivo, en transformación perpetua, que realiza operaciones de movimiento, traducción, transducción y metamorfosis (Scolari, 2019).

Para pensar su gestión y su propia existencia en una economía del conocimiento, muchas organizaciones continúan atrincheradas en la "zona de confort" del siglo XX. Pero, es evidente que los propios movimientos sociales se están encargando de demostrar que el interaprendizaje, la colaboración y la construcción colectiva son mucho más que ideales de la cultura digital y hoy se manifiestan como fuente de esperanza en medio del precariado generalizada en el planeta (Cortés, 2014b), precisamente porque la gestión comunicacional contiene un valor estratégico inherente. Pero eso depende de cuánto estemos dispuestos a aprender de manera permanente, y de cuánto de lo aprendido somos capaces de llevar a la práctica en apoyo, desde nuestro campo de trabajo, a la promoción y el acompañamiento del aprendizaje: tarea en la que se juega la gestión de la comunicación.

\section{Referencias bibliográficas}

Arribas, A. R., Herrera, E. \&. Pérez, R.A. (2017). Nueva teoría estratégica: repensando la estrategia desde la comunicación. Quito: CINTE/FISEC/Razón y Palabra.

Bates, T. (2001). National strategies for e-learning in post-secondary education and training. Paris: UNESCO: International Institute for Educational Planning.

Berry, D. M. (2011). The Computational Turn: Thinking About the Digital Humanities. Culture Machine, 12.

(ed) (2012). Understanding Digital Humanities. Nueva York: Palgrave.

Borgnakke, K. (2012). Challenges for the next generation in upper secondary school Between literacy, numeracy, and technacy. In Pink, W. T. (Ed.) (2012). Schools for marginalized youth: An international perspective. New York: Hampton Press. . (2009). Reflecting the Actual and the Future ICT based Strategies - Case studies in the practical learning context. Online Educa Berlin.

Castells, M. (1997). La Era de la Información. Economía, Cultura y Sociedad, 3 vols. (1. Sociedad red, 2. El poder de la identidad, 3. El fin del milenio). Madrid: Alianza Editorial. . (2000). Materials for an explanatory theory of the network society, British Journal of Sociology, 51 (1)pp. 5-24. . (2001). La Galaxia Internet. Reflexiones sobre Internet, empresa y sociedad. Barcelona: Plaza \& Janés. 
. (2006). The network society: from knowledge to policy. En G. Cardoso \& Castells, M. (2007). Los mitos de Internet, Barcelona: La Vanguardia.

. (2008). The New Public Sphere: Global Civil Society, Communication Networks, and Global Governance. The Annals of the American Academy of Political and Social Science. 616, no. 1. pp. 78-93.

Castells, M. (2006). (Ed). The network society: from knowledge to policy. Washington, DC: Center for Transatlantic Relations, Paul H. Nitze School of Advanced International Studies, Johns Hopkins University.

Chen, J. et al. (2019). Log Out! Worker Resistance Within and Against the Platform Economy. Toronto: McLuhan Centre for Culture and Technology.

Cobo, C. (2019). Acepto las Condiciones: Usos y abusos de las tecnologías digitales. Madrid: Fundación Santillana.

Couldry. \& Rodríguez. (2018). Desigualdad y luchas comunicativas en tiempos digitales: informe global por una comunicación comprometida con el progreso social. Bogotá: Fundación Friedrich Ebert.

Cortés, C. E. (2004). El poder de la tecnicidad digital y el reto de la complejidad, Signo y Pensamiento Bogotá: Universidad Javeriana, 44, Ene.-Jun. pp. 62-72.

. (2012). El Programa Conn@ct.Now en Colombia y el potencial de los medios sociales. Bogotá: Espacio Educativo p. 13.

. (2013a). De la capacitación al aprendizaje: La estrategia de aprendizaje ensanchado como herramienta para la gestión del cambio y del conocimiento en las organizaciones. Propuesta basada en la evaluación de acciones formativas y conformación de redes a distancia de la Federación Internacional de Fe y Alegría. Caracas: Fe y Alegría. . (2013b). La comunicación al ritmo del péndulo: seis décadas en busca del desarrollo. Pittsburgh (PA): Mimeo.

- (2014a). Tecnicidad digital, tecnicidad originaria y aprendizaje ensanchado: claves para la concepción y la práctica de la pedagogía en el siglo XXI. Pittsburgh (PA): mimeo.

. (2014b). Gestión del conocimiento, innovación y aprendizaje en tiempos de San Precario - Lectura del módulo 2 de la Especialización en Docencia Universitaria. Mendoza (Argentina): Universidad Nacional de Cuyo - Facultad de Filosofía y Letras.

Craig, R. T. (2009). Reflection on 'Communication Theory as a Field", Communiquer, 2 -, 2009, pp. 7-12.

De Liddo, A. \& Concilio, G. (2010). Supporting Communities of Practice by Advancing Knowledge Management between Hybrid Collaborative Environments, en: N. Karacapilidis (Ed.). Web-Based Learning Solutions for Communities of Practice: Developing virtual environments for social and pedagogical advancement. Hershey, PA: Information Science Reference.

Donsbach, W. (2012). The Identity of Communication Research. Signo y Pensamiento Bogotá: Universidad Javeriana, 60 (Ene.-Jun.),pp. 18 - 29.

ECOSOC. (2018). Estudio económico y social mundial 2018: Tecnologías de vanguardia en favor del desarrollo sostenible. Disponible en: https://www.un.org/development/desa/

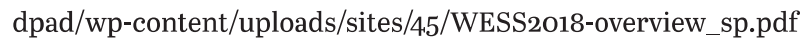

Ferreira, G. M. \&. Peruzzo, C. M. (Orgs.) (2018). Comunicação na América Latina: da metapesquisa aos estudos mediáticos. São Paulo: Intercom.

Gutiérrez, F. \& Prieto Castillo, D. (1990). La mediación pedagógica: Apuntes para una educación a distancia alternativa. San José de Costa Rica: RNTC. 
Harvey, P. L. (2017). Diseño comunáutico aplicado a los sistemas sociales digitales: fundamentos comunicacionales, teóricos y metodológicos. Bogotá: Editorial Pontificia Universidad Javeriana.

Huergo, J. (2007). Los procesos de gestión. Buenos Aires: Especialización en Política y Conducción Educativa, Universidad Pedagógica de la Provincia de Buenos Aires.

Jenkins, H. (2007). From YouTube to YouNiversity. Chronicle of Higher Education Washington, DC, 53 , no. 24.

. (2006a). Convergence Culture: Where Old and New Media Collide. Nueva York: New York University Press.

. (2006b). Confronting the Challenges of a Participatory Culture (Part Six). Disponible en: http://henryjenkins.org/blog/2006/10/confronting_the_challenges_of_5.html. Jørgensen, R. F. (2019). Human rights in the age of platforms. Cambridge, MA: The MIT Press, Kaplún, G. (2018). Investigar la comunicación en contextos de pesimismo político, incertidumbre tecnológica y voluntad democrática. En: G. M. Ferreira. \& C. M. Peruzzo (Orgs.) (2018). Comunicação na América Latina: da metapesquisa aos estudos mediáticos. São Paulo: Intercom.

Kozma, R. B. \& Isaacs, S. (2011). Transforming education: The power of ICT policies. Paris: Unesco.

Lopes, M. I. V. (2004). Pesquisa em comunicação: questões epistemológicas, teóricas e metodológicas. Revista Brasileira de Ciências da Comunicação. São Paulo: Intercom, v. XXVII, n.1, p.13-39, ene-jun.

Martín-Barbero, J.(2002a). La educación desde la comunicación. Enciclopedia latinoamericana de Sociocultura y Comunicación. Buenos Aires: Norma.

. (2002b). Tecnicidades, identidades, alteridades: des-ubicaciones y opacidades de la comunicación en el nuevo siglo, Diálogos de la Comunicación, Lima: Felafacs.

Martín Barbero, J. \& Rincón, O. (2009). Un mapa de las agendas de nación en la comunicación. En Martín Barbero, J. (Coord.). Entre saberes desechables y saberes indispensables (agendas de país desde la comunicación). Bogotá: Centro de Competencia en Comunicación para América Latina, C3 FES.

Massoni, S., Margherit, L. \& Piola, M. (2019). Investigación Enactiva en Comunicación. Estudio comparativo de proyectos caso que aplican metodología y Software IEC. RevCom, (8), eoo8. https://doi.org/10.24215/24517836eoo8.

Pérez, R. A. \& Massoni, S. (2009). Hacia una teoría general de la estrategia: El cambio de paradigma en el comportamiento humano, la sociedad y las instituciones. Barcelona: Ariel.

Peruzzo, C. M. (2018), Visões da pesquisa latino-americana em Comunicação: olhares ad-intra e ad-extra (...quem somos dentro e fora?). En: G.M. Ferreira\& C. M. Peruzzo (Orgs.) (2018). Comunicação na América Latina: da metapesquisa aos estudos mediáticos. São Paulo: Intercom, 47-78.

Piscitelli, A. (2008). La era digital y la reinvención de lo humano. En INA / RNTC (2008). Memorias del Congreso Comunicación y transmisión digital. San José de Costa Rica: INA / RNTC.

Pons, A. (2013). El desorden digital: Guía para historiadores y humanistas. Madrid: Siglo XXI.

Prieto Castillo, D. (1998). Comunicación educativa en el contexto latinoamericano, Intervención Psicosocial, Vol. 7. N. ${ }^{\circ} 3$ - pp. 329-345. (1999). La comunicación en la educación. Buenos Aires: Eds. Ciccus-La Crujía. . (2000). La enseñanza en la Universidad. 3ra. edición: Especialización en docen- 
cia universitaria. Módulo 1. Mendoza: Ediunc.

. (2002). La comunicación y la educación en el horizonte de la sociedad del conocimiento, Tegucigalpa: Reunión Unesco sobre Sociedad de la Información y la Comunicación, mimeo. . (2011), De la pedagogía a las tecnologías: En torno a las condiciones previas al trabajo a partir de competencias. Mendoza: $2^{\circ}$ Congreso Educativo Provincial, agosto. . (2013). En torno a la mayoría de edad y a la coherencia pedagógica. Palabras en el acto de inauguración de la 18a promoción de la Carrera de Especialización en Docencia Universitaria. Mendoza: Facultad de Filosofía y Letras, Universidad Nacional de Cuyo, 25 de abril.

. (2016). Notas en torno a la gestión. Borrador de trabajo para presentación oral en Curso de Gestión de la Comunicación. Maestría en Comunicación, Bogotá: UNAD.

Prieto Castillo, D. \& van de Pol, P. (2006).e-Learning, comunicación y educación: el diálogo continúa en el ciberespacio. Hilversum (Holanda) \& San José (Costa Rica): RNTC.

Rosenbloom, P. S. (2012). Towards a Conceptual Framework for the Digital Humanities. Digital Humanities Quarterly. Disponible en: http://www.digitalhumanities.org/dhq/ vol/6/2/ooo127/ooo127.html.

Rust, R. (2018). What Digital Transformation Actually Is About, The Digital Transformation People. Disponible en: https://www.thedigitaltransformationpeople.com/channels/ the-case-for-digital-transformation/digital-transformation-actually/.

Sánchez Ruiz, E. (2003). La investigación latinoamericana de la Comunicación y su entorno social: Notas para una agenda. Disponible en: http://www.comminit.com/la/ node/149928.

Schlemmer, E., D. de Q. Lopes \&. Molina, R. K. (2013). Epistemological and methodological challenges in the field of research in education and digital culture. En: F. Hernández Hernández, ,R. Fendler, , \& J. M. Sancho Gil,. (Eds.). (2013). Rethinking Educational Ethnography: Researching on-line communities and interactions. Barcelona: Universitat de Barcelona. Dipòsit Digital, 74-81. <http://hdl.handle.net/2445/44.009>

Scolari, C. (2019a). Entrevista a Néstor García Canclini. La cultura digital cambia la lectura y los modos de estudiarla. En: Varios autores (2019). Lectoescritura digital. Madrid: Ministerio de Educación y Formación Profesional. disponible en: https://sede.educacion.gob. es/publiventa/lectoescritura-digital/investigacion-educativa/22961

. (2019b). Third Law of the Interface: Interfaces form an ecosystem. Medium Technology. Disponible en: https://medium.com/@cscolari/third-law-of-the-interface-interfaces-form-an-ecosystem-e6293a108089.

Senge, P. M. (1994). The Fifth Discipline: the Art and Practice of the Learning Organization. Nueva York: Doubleday/Currency. Edición en español). La Quinta Disciplina. El arte y La práctica de las organizaciones que aprenden. Madrid: Granica.

Siapera, E. (2012). Understanding new media. Los Angeles, Calif. ; Londres: Sage.

Siemens, G. (2005). Connectivism: A Learning Theory for the Digital Age, International Journal of Instructional Technology and Distance Learning, Vol. 2 No. 1, Jan. Disponible en: http://itdl.org/journal/jan_05/articleol.htm.

The Economist (2015). Mobilegeddon: The world's biggest search engine shakes up its algorithms, The Economist, Abril 20.

Thomas, D. \& Brown, J.S. (2009). Learning for a World of Constant Change: Homo Sapiens, Homo Faber \& Homo Ludens revisited, Los Angeles: University of Southern California. 
United Nations University (2008). Innovative Capacity Development through eLearning with a Special focus on Africa. Key outcomes of our strategic UNU e-learning workshop - Bonn, pp. 26-27 November.

Van Dijck, J., Poell, T., \& Waal, M. (2018). The platform society: Public values in a connective world. Nueva York: Oxford University Press.

Vistrain, A. (2009, enero-junio). Apertura del tercer espacio y los procesos de hibridación en las situaciones de enseñanza dentro del salón de clases. CPU-e, Revista de Investigación Educativa, 8. Disponible en: <http://www.uv.mx/cpue/num8/inves/vistrain_tercer_espacio.html>.

Yang, H. H. \&. Yuen, S.C. (2010). Collective intelligence and e-learning 2.o: implications of web-based communities and networking. Hershey, PA: Information Science. 
